\title{
Ultracompact electro-optic phase modulator based on III-V-on-silicon microdisk resonator
}

\author{
J. Lloret, ${ }^{1, *}$ R. Kumar, ${ }^{2}$ S. Sales, ${ }^{1}$ F. Ramos, ${ }^{1}$ G. Morthier, ${ }^{2}$ P. Mechet, ${ }^{2}$ T. Spuesens, ${ }^{2}$ D. Van Thourhout, ${ }^{2}$ \\ N. Olivier, ${ }^{3}$ J.-M. Fédéli, ${ }^{3}$ and J. Capmany ${ }^{1}$ \\ ${ }^{1}$ Institute of Telecommunications and Multimedia Applications Research Institute, Optical and Quantum Communications Group, \\ Universitat Politècnica de València, Camino de Vera s/n, 46022 València, Spain \\ ${ }^{2}$ Department of Information Technology, Photonics Research Group, Universiteit Gent-Interuniversity MicroElectronics Center, \\ St. Pietersnieuwstraat 41, 9000 Gent, Belgium \\ ${ }^{3}$ Institute for Electronics and Information Technologies, Minatec Campus, 17 Rue des Martyrs, 38054 Grenoble, France \\ *Corresponding author: jualloso@iteam.upv.es
}

Received February 10, 2012; accepted March 12, 2012;

posted March 14, 2012 (Doc. ID 162696); published June 12, 2012

A novel ultracompact electro-optic phase modulator based on a single $9 \mu \mathrm{m}$-diameter III-V microdisk resonator heterogeneously integrated on and coupled to a nanophotonic waveguide is presented. Modulation is enabled by effective index modification through carrier injection. Proof-of-concept implementation involving binary phase shift keying modulation format is assembled. A power imbalance of $\sim 0.6 \mathrm{~dB}$ between both symbols and a modulation rate up to $1.8 \mathrm{Gbps}$ are demonstrated without using any special driving technique. () 2012 Optical Society of America

OCIS codes: $\quad 130.0250,060.5060$.

Phase modulation represents a key functionality in nextgeneration optical networks. To date, several approaches integrated on different technology platforms have been reported [1-6]. Among them, those based on silicon-oninsulator (SOI) are preferred due to the possibility of high-density integration, as well as complementary metal oxide semiconductor compatibility [7]. In silicon, phase modulation relies on controllable refractive index modification. The thermo-optic effect and the free-carrier dispersion (FCD) are the most commonly used mechanisms to implement tunable refractive index. Thermo-optics is the simplest way. However, drawbacks-such as the response times in the $\mu \mathrm{s}$ range [8], the thermal cross talk, and the large power consumption due to the continuous current flow-hamper its assembly in high-speed optical data links. On the other hand, the response time can be greatly enhanced by exploiting the FCD effect through carrier injection or depletion [7]. Carrier-depletion-based approaches can reach operation speeds of several hundreds of megabits per second. Yet large interaction lengths owing to the small light confinement in the depletion region are required. This fact in combination with an optical absorption increase provided by the carrier extraction, result in a modest power handling [9]. Regarding carrier injection, the response time is limited by the carrier recombination process in silicon, i.e., a few nanoseconds [10]. Nevertheless, the modulation efficiency is improved compared to that obtained by carrier depletion, since extra loss derived from light absorption is avoided. In order to enhance the modulation speed in FCD-based schemes, a special driving technique known as preemphasis can be used [11]. As a consequence, the speed of on-off-keyed modulated signals has been pushed up to 18 Gbps when using carrier depletion in microrings [12]. Aiming at improving the sensitivity and robustness against nonlinear effects, advanced modulation formats can be implemented. Recently, error-free transmission of differential-phase-shift-keyed modulated signals at
5 Gbps exploiting carrier depletion with preemphasis in silicon microrings has been demonstrated [13].

Hybrid approaches consisting of bonding active III-V materials on top of SOI circuits represent a feasible solution in order to enhance the modulating performance $[14,15]$. Inherent dynamics in semiconductor materials is faster than in silicon. In particular, carrier depletion has been used in a $1 \mathrm{~mm}$-long hybrid silicon-AlGaInAs waveguide. The speed is not further limited by the carrier dynamics but by parasitic capacitance in the metal contacts [15]. The bandwidth can be extended to few tens of gigabits per second assisted by an optimized microwave design [14].

In this Letter, we propose a novel hybrid approach based on a single InP microdisk resonator (MDR) heterogeneously integrated on and coupled to a nanophotonic SOI waveguide circuit containing a $650 \mathrm{~nm}$-wide and $220 \mathrm{~nm}$-high Si strip waveguide using molecular bonding. The $9 \mu$ m-diameter MDR includes three strained InPInGaAsP quantum wells and a tunnel junction for loss minimization purposes. The propagation is based on whispering-gallery modes, which are confined to the edges of the MDR resulting in a resonant-type response. More details on the design and fabrication of such a device are given in $[16,17]$. This proposal is low powerconsuming and more compact compared to [15]. Similar to $[\underline{14}, 15]$, the operating speed is at the end limited by $R C$ effects in the metal contacts. Purely resistive behavior could enable modulation speeds in the order of several tens of gigabits per second without any special driving technique. Besides, preemphasis could be also exploited to expand the modulation rate. By using the MDR, amplitude modulation enabled by carrier injection has already been demonstrated [18].

The experimental setup is sketched in Fig. 1(a). TEpolarized light from a tunable laser operated in continuous wave $(\mathrm{CW})$ was coupled into the SOI waveguide. The modulating signal was generated by a pattern generator in combination with a direct current (DC) voltage source. 


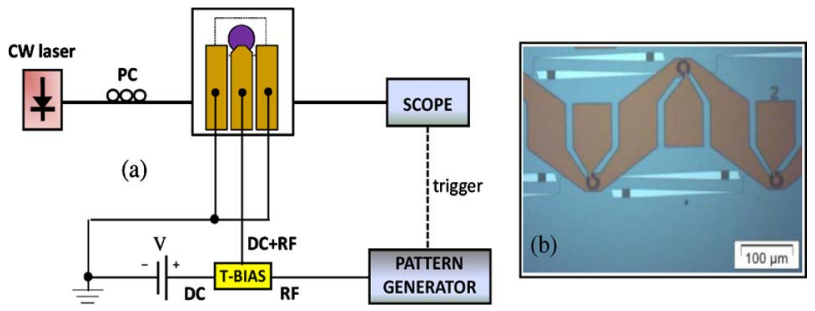

Fig. 1. (Color online) (a) Sketch showing the experimental setup, (b) top-view micrograph of fabricated MDRs.

At the MDR output, the light was collected using a highbandwidth oscilloscope. Figure 1(b) depicts a footprint image of the device, showing the metal contacts and the input and output vertical grating couplers.

The principle of operation focuses on exploiting the phase change that occurs in the vicinity of a resonance under overcoupled regime. This phase change is controlled by the effective index modification through carrier injection. Figure 2 shows both the gain and phase transfer functions with the current injected into the MDR. The spectral position of the notch can be modified by changing the injection current. Consequently, the phase response is accordingly shifted. As a result, the phase experienced by the optical carrier can be adjusted by means of the injection current. On one hand, the MDR must be operated below threshold to avoid coherencyrelated problems. Otherwise, the lasing mode appearing in the resonance would beat with the optical carrier resulting in distortion of the modulated output signal. This fact limits the maximum injection current. On the other hand, the overcoupled regime is required to obtain full $360^{\circ}$ phase shifts at the notch position. To guarantee overcoupling, the roundtrip loss must be lower than the coupling factor. Hence, a minimum current yielding high enough gain in the III-V compound must be injected.

The performance of the phase modulator was evaluated when considering binary phase shift keying (BPSK) modulation. The emission wavelength of the optical carrier was set to $1561.815 \mathrm{~nm}$ in order to achieve $180^{\circ}$ phase shift when sweeping the injection current into the MDR from 1 to $1.2 \mathrm{~mA}$ (inset of Fig. 2). This
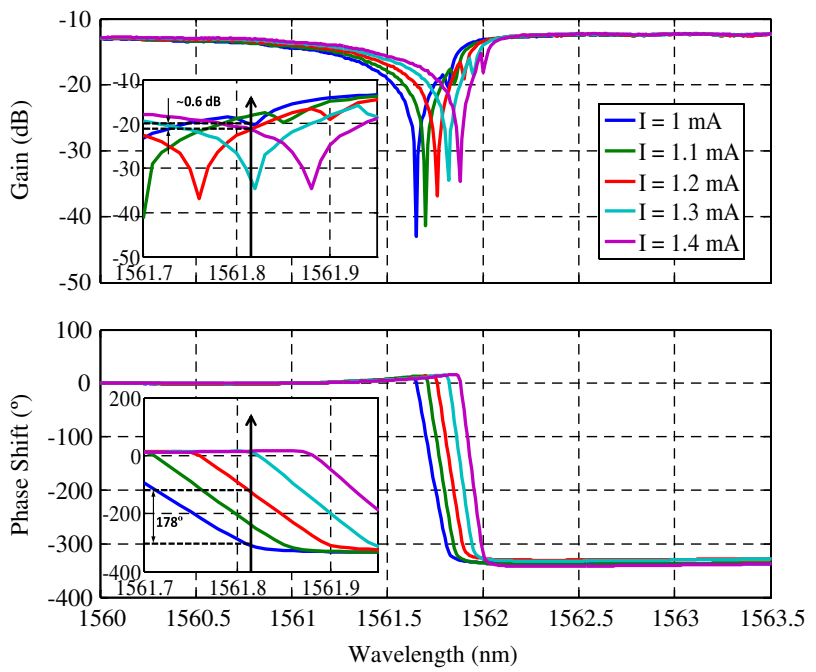

Fig. 2. (Color online) Gain and phase transfer functions of the MDR and detail of the optical carrier spectral placement.
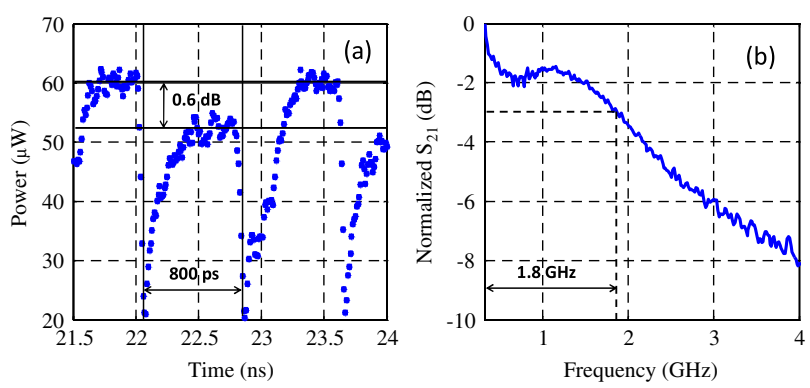

Fig. 3. (Color online) (a) Waveform at the MDR output, (b) small-signal response, $S_{21}$, of the MDR.

wavelength was also determined by the gain response, since power variations as low as possible within the current range were desired. A power difference between both modulation symbols of only $0.6 \mathrm{~dB}$ was obtained, as illustrated in the inset of Fig. 2. The output power of the CW laser was set to $2 \mathrm{dBm}$. As a proof-of-concept, a 127 bit nonreturn to zero (NRZ) square-wave signal at 1.25 Gbps was applied at the metal contacts. The DC source was set to a voltage resulting in $1 \mathrm{~mA}$ bias current delivered, while the peak-to-peak level of the NRZ signal was $0.57 \mathrm{~V}$, corresponding to $200 \mathrm{~mA}$.

Figure 3(a) shows the waveform at the MDR output. After reaching the steady state, a power imbalance of nearly $0.6 \mathrm{~dB}$ between both levels was measured. This is in perfect agreement with the results derived from Fig. 2. From the output waveform, the rise and fall times can be estimated, being $\sim 400$ and $\sim 150 \mathrm{ps}$, respectively. Consequently, the maximum modulation speed is estimated as approximately 1.8 Gbps. The small-signal response of the MDR, $S_{21}$, is displayed in Fig. 3(b). An injection current of $1 \mathrm{~mA}$ and a $\mathrm{CW}$ laser output power of $2 \mathrm{dBm}$ were used. The $-3 \mathrm{~dB}$ bandwidth was around $1.8 \mathrm{GHz}$. This confirms that modulation rates as high as 1.8 Gbps could be achieved, as previously derived from the output waveform.

Next, the phase shift of the modulated output signal was investigated. For this purpose, a Mach-Zehnder interferometer (MZI) was used with the aim at converting phase-to-intensity modulation (PM-IM). The setup is illustrated in Fig. 4. A $4 \mathrm{dBm}$-power optical signal at $1561.815 \mathrm{~nm}$ was split employing a $3 \mathrm{~dB}$ coupler. In the lower arm, the MDR-based phase modulator was inserted. The length of both branches was perfectly balanced by means of a variable delay line (VDL). The

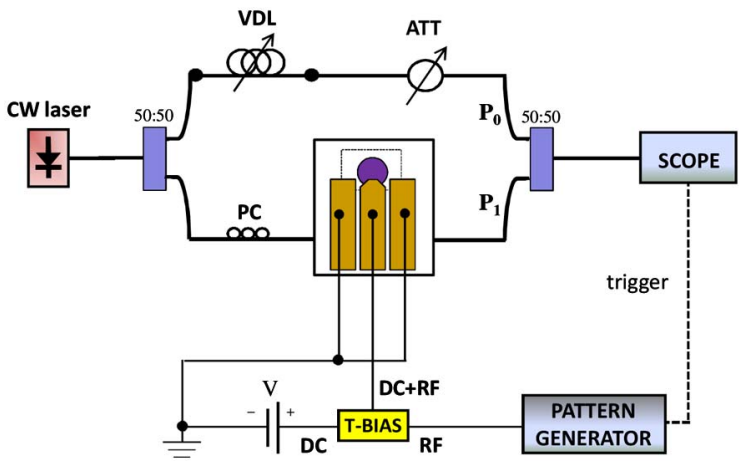

Fig. 4. (Color online) Interferometric-based setup for PM-IM conversion. 


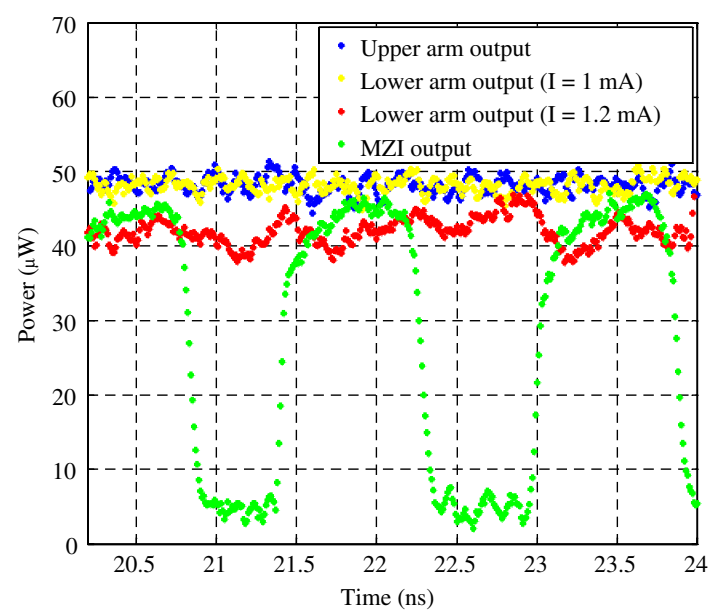

Fig. 5. (Color online) Waveforms at the MZI output and at the upper and lower arm outputs prior to the coupler.

coupled output signal was collected by the oscilloscope. A variable attenuator was also used in the upper arm to compensate the loss derived from the MDR.

Results are displayed in Fig. $\underline{5}$. The optical power was measured prior to the output coupler in both arms under static conditions. These powers are named as $P_{0}$ and $P_{1}$ in Fig. 4, respectively. The attenuator was adjusted for obtaining the same average power level at the upper and lower arm outputs when considering an injection current of $1 \mathrm{~mA}$. The powers $P_{0}$ and $P_{1}$ were around $48.2 \mu \mathrm{W}$ and the attenuation was fixed to $11.6 \mathrm{~dB}$. For the lower arm, $P_{1}$ was also measured when injecting $1.2 \mathrm{~mA}$. The average power difference was around $0.6 \mathrm{~dB}$. This value perfectly agrees with the preceding results. Following, dynamic measurements were accomplished by applying the same driving signal that was previously used. When $P_{0}$ and $P_{1}$ are identical, and the phase imbalance between both arms is null, constructive interference takes place at the MZI output. This means that the total output power is equal to $P_{0}$ or $P_{1}$. Conversely, phase imbalance of $180^{\circ}$ yields destructive interference, giving as a result no power at the output. The MZI output power is also shown in Fig. 5. An extinction ratio of $10.3 \mathrm{~dB}$ was obtained, demonstrating the suitability of the MDR to be operated as an electro-optic phase shifter. Larger extinction ratio was not reached as a consequence of the power deviation between both modulation symbols and spontaneous noise generated by the MDR.

In conclusion, a novel electro-optic phase modulator based on III-V-on-silicon MDR has been introduced. Carrier injection enables effective index modifications resulting in phase modulation. Proof-of-concept implementation involving BPSK modulation at $1.25 \mathrm{Gbps}$ with a power imbalance of $\sim 0.6 \mathrm{~dB}$ between both symbols has been demonstrated. A peak-to-peak voltage of just $0.57 \mathrm{~V}$ is needed for the modulating signal. The modulation rate could be pushed up to $1.8 \mathrm{Gbps}$ without using any special driving technique according to the time and frequency MDR responses. Furthermore, this modulation rate can be extended to several tens of gigabits per second assisted by an optimized microwave design in order to minimize the parasitic effects in the metal contacts.

The authors gratefully acknowledge the financial support given by the European Seventh Framework Programme through the projects GOSPEL, WADIMOS, and HISTORIC; the Generalitat Valenciana through the Microwave Photonics Excellency Award programme GVA PROMETEO 2008/092; and also the Plan Nacional I+D TEC2011-29120-C05-05 and TEC2008-06145.

\section{References}

1. E. L. Wooten, K. M. Kissa, A. Yi-Yan, E. J. Murphy, D. A. Lafau, P. F. Hallemeier, D. Maack, D. V. Attanasio, D. F. Fritz, G. J. McBrien, and D. E. Bossi, IEEE J. Sel. Top. Quantum Electron. 6, 69 (2000).

2. Y. Li, R. Wang, A. Bhardwaj, S. Ristic, and J. Bowers, IEEE Photon. Technol. Lett. 22, 1340 (2010).

3. C. E. Png, S. P. Chan, S. T. Lim, and G. T. Reed, IEEE J. Lightwave Technol. 22, 1573 (2004).

4. W.-C. Chiu, C.-C. Chang, J.-M. Wu, M.-C. M. Lee, and J.-M. Shieh, Opt. Lett. 36, 1089 (2011).

5. Y.-H. Lin and Y.-S. Tsou, J. Appl. Phys. 110, 114516 (2011).

6. S.-K. Kim, W. Yuan, K. Geary, Y.-C. Hung, H. R. Fetterman, D.-G. Lee, C. Zhang, C. Wang, W. H. Steier, G.-C. Park, S.-J. Kang, and I. Oh, Appl. Phys. Lett. 87, 011107 (2005).

7. R. A. Soref and B. R. Bennet, IEEE J. Quantum Electron. 23, 123 (1987).

8. R. L. Espinola, M. C. Tsai, J. T. Yardley, and R. M. Osgood, IEEE Photon. Technol. Lett. 15, 1366 (2003).

9. N. N. Feng, S. R. Liao, D. Z. Feng, P. Dong, D. W. Zheng, H. Liang, R. Shafiiha, G. L. Li, J. E. Cunningham, A. V. Krishnamoorthy, and M. Asghari, Opt. Express 18, 7994 (2010).

10. Q. Xu, B. Schmidt, S. Pradhan, and M. Lipson, Nature 435 , 325 (2005).

11. Q. Xu, S. Manipatruni, B. Schmidt, J. Shakya, and M. Lipson, Opt. Express 15, 430 (2007).

12. S. Manipatruni, Q. Xu, B. Schmidt, J. Shakya, and M. Lipson, in Proceedings of the 20th Annual Meeting of the IEEE Lasers and Electro-Optics Society, 2007 (IEEE, 2007), paper WO2.

13. K. Padmaraju, N. Ophir, Q. Xu, B. Schmidt, J. Shakya, S. Manipatruni, M. Lipson, and K. Bergman, in Proceedings of the 2011 37th European Conference and Exhibition on Optical Communications (ECOC) (IEEE, 2011), paper Th. 12 .

14. Y.-H. Kuo, H.-W. Chen, and J. E. Bowers, Opt. Express 16, 9936 (2008).

15. H.-W. Chen, Y.-H. Kuo, and J. E. Bowers, IEEE Photon. Technol. Lett. 20, 1920 (2008).

16. J. Van Campenhout, P. Rojo-Romeo, D. Van Thourhout, C. Seassal, P. Regreny, L. Di Cioccio, J.-M. Fedeli, and R. Baets, IEEE J. Lightwave Technol. 26, 52 (2008).

17. D. Van Thourhout, T. Spuesens, S. K. Selavaraja, L. Liu, G. Roelkens, R. Kumar, G. Morthier, P. Rojo-Romeo, F. Mandorlo, P. Regreny, O. Raz, C. Kopp, and L. Grenouillet, IEEE J. Sel. Top. Quantum Electron. 16, 1363 (2010).

18. L. Liu, J. Van Campenhout, G. Roelkens, R. A. Soref, D. Van Thourhout, P. Rojo-Romeo, P. Regreny, C. Seassal, J.-M. Fedeli, and R. Baets, Opt. Lett. 33, 2518 (2008). 